\title{
Trans Disciplinary Biomedical Research
}

\author{
Prof. Mohamed Labib Salem
}

Prof. of Immunology, Faculty of Science, Tanta University, Egypt

Editor-in-Chief, JCBR

E-mail: eacr@unv.tanta.edu.eg

Recently, academicians and clinicians have been focusing their researches on solving scientific challenges meetings the medical and related biological sciences using technologies that were developed in the last decades. These researches has led to a growing body of scientific data in the field of biomedical research that led to breakthrough discoveries in health and disease in particular cancer. These discoveries are based on inter-, multi-, and trans-disciplinary approaches. Although both multi- and interdisciplinary research in the last decades have led to major findings with paramount significance in translational research from bench to clinic, the coming era requires more trans disciplinary research based on sophisticated networking between researchers from different disciplines. Ultimately, multidisciplinary Research draws on knowledge from different disciplines but stays within their boundaries. Interdisciplinary research analyzes, synthesizes and harmonizes links between disciplines into a coordinated and coherent whole. Trans disciplinary research integrates the natural, social and health sciences in a humanities context, and transcends their traditional boundaries.

Indeed, trans disciplinary research requires three approaches of leadership. The first is the effective structural leadership, which adds value by creating needed bridges among unconnected parties effective cognitive leadership, which provides a vision that links and motivates trans disciplinary researchers to step beyond their disciplinary lens, relax old assumptions, and search for creative framebreaking solutions. The second is Effective structural leadership, which adds value by creating needed bridges among unconnected parties. The third is the effective processual leadership, which encourages trust and turns potentially destructive conflict into constructive

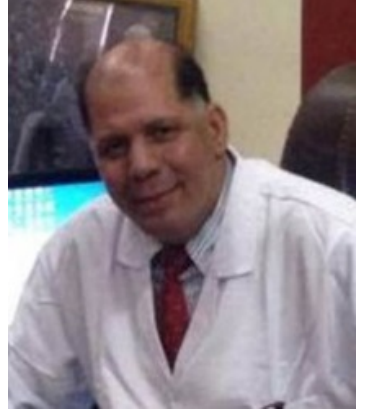

interactions. Because, in most developing countries trans disciplinary research is rare, cutting-edge research is low as indicated to the few numbers of original articles published in the top peer-reviewed journals with high impact factors.

In the Middle East countries, few journals cover the gap between basic biological sciences and translational biomedical sciences. As such, we thought to issue the International Journal of Cancer and Biomedical Research (IJCBR) as a new platform to cover such two major disciplinarians. This journal will focus on fostering such gap through focusing on interdisciplinary studies. By having this journal, I would expect emergence of publication of several studies with paramount significance to the advancement of cutting-edge research based on basic and recent technologies including single cell analysis, proteomics and genomic arrays. As an editor to the JCBR, I am confident that this journal will be spread widely in the coming years to cover all topics in both cancer and biomedical subjects.

\section{REFERENCES}

Brandt P, Ernst A, Gralla F, Luederitz C, Lang D, Newig J, Reinert F, Abson J and Wehrden H (2013) A review of transdisciplinary research in sustainability science. Ecological Economics; 92: 1-15.

Bracken LJ, Bulkeley HA and Whitman G. (2015) Transdisciplinary research: understanding the stakeholder perspective, Journal of Environmental Planning and Management; 58(7): 12911308. 